\title{
Circulating plasma exosomes in obstructive sleep apnoea and reverse dipping blood pressure
}

\author{
Abdelnaby Khalyfa $\mathbb{0}^{1}$, David Gozal $\mathbb{1}^{1}$, Wen-Ching Chan², Jorge Andrade ${ }^{2}$ and \\ Bharati Prasad ${ }^{3}$
}

Affiliations: ${ }^{1}$ Dept of Child Health and the Child Health Research Institute, University of Missouri School of Medicine, Columbia, MO, USA. ${ }^{2}$ Center for Research Informatics, The University of Chicago, Chicago, IL, USA. ${ }^{3}$ Division of Pulmonary, Critical Care, Sleep and Allergy, Dept of Medicine, University of Illinois at Chicago and Jesse Brown VA Medical Center, Chicago, IL, USA.

Correspondence: Bharati Prasad, Division of Pulmonary, Critical Care, Sleep, and Allergy, Dept of Medicine, University of Illinois Hospital and Health Sciences System, 840 S. Wood Street, MC 719, 9th Floor, Chicago, IL 60612, USA. E-mail: bpradsadquic.edu

@ERSpublications

A selected number of circulating exosomal miRNAs play an important role in abnormal circadian regulation of blood pressure and may provide prognostic biomarkers of cardiovascular risk in OSA http://bit.ly/2VPRFl4

Cite this article as: Khalyfa A, Gozal D, Chan W-C, et al. Circulating plasma exosomes in obstructive sleep apnoea and reverse dipping blood pressure. Eur Respir J 2020; 55: 1901072 [https://doi.org/10.1183/ 13993003.01072-2019].

\section{ABSTRACT}

Background: Obstructive sleep apnoea (OSA) increases the risk of an abnormal nondipping $24 \mathrm{~h}$ blood pressure profile, an independent risk factor for cardiovascular disease (CVD). We examined differential exosomal microRNA (miRNA) expression in untreated OSA patients with normal dipping blood pressure (NDBP) and reverse dipping blood pressure (RDBP), an extreme form of nondipping, to understand the mechanisms underlying nondipping blood pressure in OSA.

Methods: 46 patients (15 RDBP versus $31 \mathrm{NDBP}$ ) matched for OSA severity (respiratory event index $32.6 \pm 22.5$ versus $32.2 \pm 18.1$ events $\left.\cdot \mathrm{h}^{-1} ; \mathrm{p}=0.9\right)$, age $(54.8 \pm 12.9$ versus $49 \pm 9.9$ years; $\mathrm{p}=0.09)$ and body mass index $\left(36.2 \pm 6.6\right.$ versus $\left.34.4 \pm 6.8 \mathrm{~kg} \cdot \mathrm{m}^{-2} ; \mathrm{p}=0.4\right)$ were included. Plasma exosomes were characterised by flow cytometry and functional in vitro reporter assays were conducted on cultured endothelial cells. Exosome miRNA cargo was profiled with microarrays followed by bioinformatics analyses.

Results: Exosomes from RDBP patients increased the permeability of endothelial cell tight junctions and adhesion molecule expression. Principal component analyses of miRNA array data showed strict separation and identification of the two groups. A restricted and validated signature of exosomal miRNAs was identified in the RDBP versus NDBP group. Their predicted target genes involved phosphatidylinositol 3-kinase-Akt ( $\mathrm{p}=0.004)$, Ras $(p=3.42 E-05)$, Wnt $(p=0.003)$ and hypoxia inducible factor- 1 signalling $(p=0.04)$, inflammatory mediator regulation of transient receptor potential channels $(\mathrm{p}=0.01)$, and several cancer-related pathways.

Conclusions: Patients with RDBP have altered miRNA cargoes in circulating exosomes that invoke in vitro endothelial dysfunction. A selected number of circulating exosomal miRNAs play an important role in abnormal circadian regulation of blood pressure and may provide prognostic biomarkers of CVD risk in OSA.

This article has supplementary material available from erj.ersjournals.com

This study is registered at ClinicalTrials.gov with identifier number NCT01960465. De-identified data will be shared upon request.

Received: 30 May 2019 | Accepted after revision: 12 Oct 2019

Copyright $\odot$ ERS 2020 


\section{Introduction}

Obstructive sleep apnoea (OSA) is characterised by intermittent hypoxia and sleep fragmentation [1], and promotes the abolition of the normal nocturnal blood pressure decline that occurs during sleep [2-4]. The emergence of a nondipping blood pressure phenomenon is an independent predictor of organ damage and cardiovascular disease (CVD) $[5,6]$. Abnormal circadian patterns of blood pressure in OSA range from nondipping $(<10 \%$ nocturnal decline) to reverse dipping, where a rise, rather than a decline, in average nocturnal blood pressure occurs compared with average diurnal values.

The mechanisms underlying hypertension and nondipping in OSA include increased sympathetic nervous system activity and endothelial dysfunction $[7,8]$. In particular, endothelial dysfunction in OSA is an early event preceding the clinical manifestations of CVD $[9,10]$. Therefore, examining the molecular basis of nondipping in clinical populations with OSA and hypertension and the potential effects on endothelial function can improve our understanding of the mechanistic underpinnings of OSA-related hypertension.

Exosomes are 30-100 $\mathrm{nm}$ extracellular vesicles secreted by cells into the circulation [11-13]. Exosomes carry a biologically active cargo derived from parent cells, which depends on the status of the parent cells at the time of exosome biogenesis [14-16]. Circulating exosomes are captured by acceptor cells, where the exosomal microRNAs (miRNAs), small noncoding RNAs, can regulate gene expression post-transcriptionally, thus acting as vehicles for intercellular communication [17]. The diverse exosomal cargo, which consists of miRNAs, proteins, lipids, DNA and RNA, provides unique opportunities for biomarker discovery and development of noninvasive diagnostic approaches using urine or blood [12, 14, 18]. We have previously shown that exosomal miRNAs play a critical role in multiple cardiometabolic diseases [19-21]. Furthermore, exosomes shed by endothelial cells are involved in CVD [22].

Here, we examined exosomal miRNA cargo in a cohort of untreated OSA patients with hypertension. First, differences in the expression of exosomal miRNAs were identified between two groups of patients within this cohort: those with an abnormal circadian profile on $24 \mathrm{~h}$ ambulatory blood pressure (ABP) recordings (reverse dipping blood pressure (RDBP)) compared with a matched group with a normal dipping blood pressure (NDBP) profile. Next, we examined the functional effects of differentially expressed exosomal miRNA on endothelial function by a series of in vitro reporter assays, as well as potential differences in exosomal miRNA content between RDBP and NDBP. We hypothesised that differences in exosomal miRNA expression and their functional implications would identify genomic pathways underlying endothelial dysfunction, an early and essential mechanism of OSA-related hypertension.

\section{Material and methods \\ Participants}

The study sample was a subset of participants drawn from a prospective study $(n=160)$ to examine race and sleepiness as predictors of hypertension in OSA. 15 participants in this study were identified to have $\mathrm{RDBP}$ (i.e. a rise in blood pressure during sleep compared with waking values) on $24 \mathrm{~h}$ ABP monitoring. They were matched to 31 participants with normal dipping ABP (NDBP) from the same study based on age, OSA severity and body mass index (BMI). Participants were between 18 and 70 years of age, with hypertension and untreated moderate-to-severe OSA. Hypertension was defined as a physician diagnosis, use of antihypertensive medications or repeated office blood pressure $\geqslant 140 / 90 \mathrm{mmHg}$ within 3 months of enrolment. Home sleep testing (Alice NightOne type 3; Philips North America, Andover, MA, USA) was used to diagnose OSA. A home sleep test was deemed acceptable if the recording was at least $3 \mathrm{~h}$ in duration with functional oximetry, respiratory effort and airflow channels. OSA was defined by a respiratory event index $(\mathrm{REI}) \geqslant 15$ events $\mathrm{h}^{-1}$. Home sleep tests were manually scored by a board-certified sleep physician. Apnoeas were scored for flow reduction $>90 \%$ for $\geqslant 10$ s. Hypopnoeas were scored by $\geqslant 30 \%$ reduction in flow associated with $\geqslant 3 \%$ oxygen desaturation. Participants with $\geqslant 25 \%$ central or mixed apnoeas were excluded. Participants completed the Epworth Sleepiness Scale, 3-7 days of wrist actigraphy (Actiwatch 2; Philips Respironics), ABP monitoring and $24 \mathrm{~h}$ urine collection for catecholamines. ABP measurements were taken every $20 \mathrm{~min}$ during the day and every $30 \mathrm{~min}$ at night, using a Spacelabs 90217 (Spacelabs Medical, Snoqualmie, WA, USA) ABP monitor. Urine catecholamines were measured with quantitative high-performance liquid chromatography-tandem mass spectrometry (ARUP Laboratories, Salt Lake City, UT, USA). Participants on medications that may affect urine catecholamine assays were excluded. Participants on diuretics had normal serum sodium levels. ABP recordings were deemed acceptable if $>70 \%$ of measurements were completed. Dipping was calculated by the Spacelabs software using: average wake ABP-average sleep ABP/average wake ABP. Dipping status was determined by mean ABP (mean blood pressure=systolic blood pressure $+2 \times$ diastolic blood pressure $/ 3$ ). Exclusion criteria included previous medical or surgical treatment of OSA, uncontrolled medical or psychiatric disease, hospitalisation within 3 months of enrolment, shift work, occupational driving and 
pregnancy. Phlebotomy to collect plasma samples was conducted in the morning. The experimental procedures are shown in figure 1.

This study was conducted per the amended Declaration of Helsinki, and University of Illinois at Chicago (20131086-81081) and Jesse Brown VA (756434-2) Institutional Review Board approvals. Written informed consent was obtained from all patients.

\section{Exosome isolation, characterisation and functional effects on endothelial cells}

Exosomes samples were stored at $-20^{\circ} \mathrm{C}$ for short-term use and stored long term at $-80^{\circ} \mathrm{C}$ in several aliquots in top-cap tubes. Exosome isolation, size determination and quantification were performed by methods previously reported and detailed in the supplementary material [19, 23, 24]. In vitro materials and methods used to examine differences in exosomes properties and miRNA cargo between RDBP and NDBP groups included: 1) endothelial cell culture (human dermal microvascular endothelial cell (hMVEC-d) monolayer) [20, 25], 2) exosome characterisation by flow cytometry, 3) exosome uptake by endothelial cells using fluorescent linker PKH26-Red [26], and 4) endothelial monolayer barrier function by i) electric cell-substrate impedance sensing (ECIS) [25], ii) trans-endothelial electric resistance (TEER) [27], and iii) examination of tight junctions and adherens junction protein and glycoproteins, namely zonula occludens (ZO)-1, VE-cadherin and intercellular adhesion molecule (ICAM)-1, by immunofluorescence, confocal microscopy and stimulated emission depletion (STED) super-resolution microscopy, respectively. All of the materials and methods are detailed in the supplementary material.

\section{Exosomal miRNA isolation and microarrays}

Total RNA, including miRNA, was extracted from isolated exosomes using a miRNeasy Mini Kit column-based system (Qiagen, Valencia, CA, USA) following the manufacturer's instructions as previously described [20]. Microarray results were extracted using Agilent Feature Extraction version 12.0 [19, 28] to quantify signal intensities. The quality control for each miRNAs was evaluated based on their Agilent Feature Extraction quality control report (supplementary material). Functional annotation of differentially expressed miRNA, miRNA target gene predictions, regulatory network construction and quantitative real-time (qRT)-PCR validation of microarray results are further described in the supplementary material.

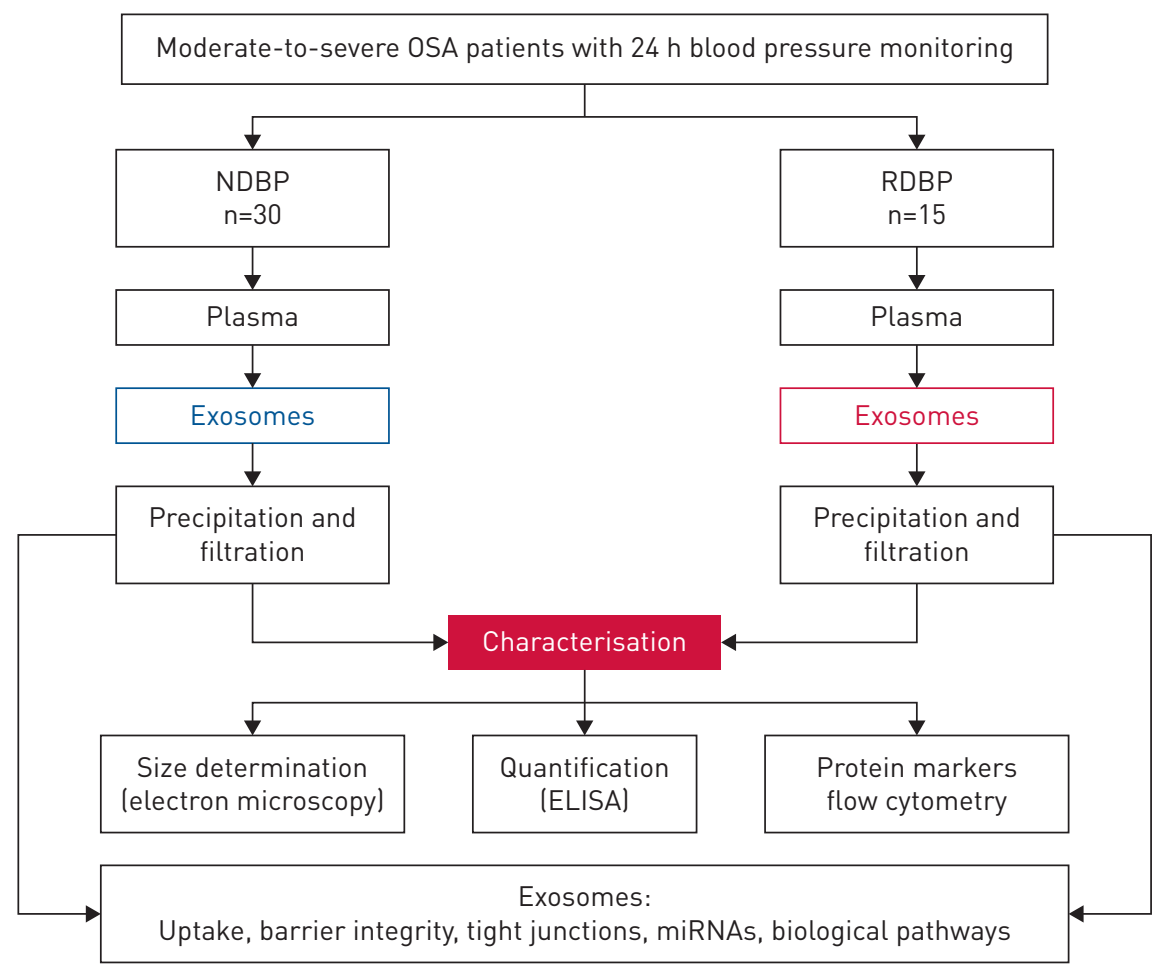

FIGURE 1 Schematic diagram illustrating the experimental design and data analyses. OSA: obstructive sleep apnoea; NDBP: normal dipping blood pressure; RDBP: reverse dipping blood pressure; miRNA: microRNA. Plasma exosomes were isolated from each of the matched subjects with OSA and either NDBP or RDBP patterns during sleep. 
Data analysis

This proof-of-concept study was performed on a subset of matched patients derived from a cohort study. No a priori power calculation was performed. All data are expressed as mean with standard deviation, unless otherwise stated. All numerical data were subjected to statistical analysis using paired t-tests or ANOVA followed by post hoc tests (Tukey) as appropriate. Chi-squared analysis was performed on categorical data concerning demographic characteristics of the various groups. All analyses were conducted using SPSS version 21.0 (IBM, Armonk, NY, USA). Comparisons between exosome-treated and untreated cells were performed using t-tests or one-way ANOVA with Bonferroni's multiple comparison tests. $\mathrm{p}<0.05$ was considered statistically significant.

\section{Results}

\section{Patient characteristics}

46 patients were classified into two groups (RDBP $n=15$ versus NDBP $n=31$ ) matched by OSA severity

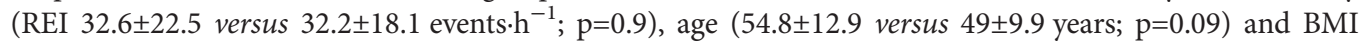
$\left(36.2 \pm 6.6\right.$ versus $34.4 \pm 6.8 \mathrm{~kg} \cdot \mathrm{m}^{-2}$; $\left.\mathrm{p}=0.4\right)$ as described in table 1 .

\section{Exosome characterisation and quantifications}

Plasma exosome isolation, characterisation and quantification derived from RDBP are shown in supplementary figure S1. Confirming published results [19], negative stain electron microscopy showed vesicles in typically shaped morphology (supplementary figure S1a). Supplementary figure S1b shows no difference in the quantification of exosomes derived from NDBP $\left(4.44 \times 10^{8} \mathrm{~mL}^{-1}\right)$ versus RDBP $\left(4.50 \times 10^{8} \mathrm{~mL}^{-1}\right)$. To determine unique markers specific to exosomes of purified samples, different exosome markers were used, including tetraspanins (CD63; exosome formation and secretion), targeting/ adhesion (CD31; exosome maturation and target cell binding) and membrane transport (Rab5b; exosome biogenesis, secretion and cell fusion), as shown in supplementary figure S1c. Flow cytometry of isolated exosomes from NDBP or RDBP groups revealed the presence of tetraspanins, targeting/adhesion and antigen presentation markers as anticipated from highly purified (>96\%) exosome fractions (supplementary figure S1c).

TABLE 1 Demographic characteristics of subjects with normal dipping blood pressure (NDBP) and reverse dipping blood pressure (RDBP) recruited and included in the study

\begin{tabular}{|c|c|c|c|}
\hline & NDBP & RDBP & p-value \\
\hline Subjects $\mathrm{n}$ & 31 & 15 & \\
\hline Age years & $49 \pm 9.9$ & $54.8 \pm 12.9$ & 0.09 \\
\hline \multicolumn{4}{|l|}{ Race $n$} \\
\hline African-American & 18 & 10 & 0.6 \\
\hline Caucasian & 8 & 3 & \\
\hline Other & 5 & 2 & \\
\hline Male & 27 & 14 & 0.5 \\
\hline BMI $\mathrm{kg} \cdot \mathrm{m}^{-2}$ & $34.4 \pm 6.8$ & $36.2 \pm 6.6$ & 0.4 \\
\hline REI events $\cdot h^{-1}$ & $32.6 \pm 22.5$ & $32.2 \pm 18.1$ & 0.9 \\
\hline $0 D I 3$ events $\cdot h^{-1}$ & $28.7 \pm 18.9$ & $28.5 \pm 23.0$ & 0.9 \\
\hline Nadir $S_{\mathrm{po}_{2}} \%$ & $77.2 \pm 1.6$ & $78.4 \pm 1.6$ & 0.6 \\
\hline$T<90$ min & $81.3 \pm 95.9$ & $46.6 \pm 50.1$ & 0.2 \\
\hline ESS & $11.2 \pm 5.0$ & $12.5 \pm 4.9$ & 0.4 \\
\hline TST h & $6.0 \pm 1.7$ & $5.2 \pm 1.6$ & 0.1 \\
\hline Systolic ABP mmHg & $127.7 \pm 10.53$ & $130.3 \pm 12.6$ & 0.4 \\
\hline Systolic ABP dipping $\mathrm{mmHg}$ & $0.14 \pm 0.03$ & $-0.07 \pm 0.05$ & $<0.05^{\#}$ \\
\hline Diastolic ABP mmHg & $78.2 \pm 8.8$ & $77.9 \pm 7.2$ & 0.9 \\
\hline Diastolic ABP dipping $\mathrm{mmHg}$ & $0.17 \pm 0.03$ & $-0.10 \pm 0.07$ & $<0.05^{\#}$ \\
\hline Mean ABP $\mathrm{mmHg}$ & $93.8 \pm 7.85$ & $94.8 \pm 7.11$ & 0.7 \\
\hline Mean ABP dipping $\mathrm{mmHg}$ & $0.15 \pm 0.03$ & $-0.08 \pm 0.05$ & $<0.05^{\#}$ \\
\hline Urine epinephrine $\mu \mathrm{g} \cdot 24 \mathrm{~h}^{-1}$ & $5.5 \pm 2.7$ & $6.0 \pm 3.9$ & 0.6 \\
\hline Urine norepinephrine $\mu \mathrm{g} \cdot 24 \mathrm{~h}^{-1}$ & $56.7 \pm 26.7$ & $71.5 \pm 45.8$ & 0.1 \\
\hline
\end{tabular}

Data are presented as mean $\pm \mathrm{SD}$, unless otherwise stated. BMI: body mass index; REI: respiratory event index; ODI3: $3 \%$ oxygen desaturation index; $\mathrm{S}_{\mathrm{pO}_{2}}$ : arterial oxygen saturation measured by pulse oximetry; $T<90$ : time < $90 \% \mathrm{~S}_{\mathrm{pO}_{2}}$; ESS: Epworth Sleepiness Scale; TST: total sleep time; ABP: ambulatory mean blood pressure. ${ }^{\#}$ : statistically significant. 


\section{Exosomes uptake using PKH26}

To test whether exosomes isolated from the RDBP and NDBP groups were incorporated into human naive endothelial cells, the culture media of the cells was supplemented individually with PKH26-labelled exosomes (figure 2). Endothelial uptake of exosomes from NDBP and RDBP was similar for the six patients tested from each group. The PKH26 signal was observed in the lipid cell membrane of cells grown in medium supplemented with PKH26-labelled exosomes, whereas no signal was observed in cells grown in medium supplemented without exosomes to which PKH26 was also added. After validation of the quality of purified exosome samples using known exosomal markers, we determined the bioactivity of exosomes by examining cell uptake after the isolation procedures (figure 2). In this setting, cells exhibiting exosome uptake showed that both RNA and protein colocalised with 4',6-diamidino-2-phenylindole in the nucleus, while lipid localisation was restricted to the cell membrane. Exosome uptake was dose dependent, as there were increased spot numbers, total fluorescence and median pixel intensities of the dyes with increasing exosome concentrations (data not shown).

\section{ECIS experiments}

Exosomes from RDBP patients induced greater disruption of the endothelial monolayer barrier integrity compared with NDBP patients (figure $3 \mathrm{a}$ and $\mathrm{b}$ ). The normalised resistance change was higher in the RDBP group $(-78 \pm-7.2 \%)$ compared with the NDBP group $(-57 \pm-5.3 \%)(\mathrm{p}=0.001)$ (figure $3 \mathrm{~b})$.

TEER is a sensitive method to confirm the integrity and permeability of the cell monolayer. Increased TEER is directly correlated with decreased endothelial cell paracellular permeability [29, 30]. We found that the TEER values in transwells treated with exosomes derived from RDBP were $3.61 \pm 0.13 \Omega \cdot \mathrm{cm}^{-2}$ compared with $6.61 \pm 0.57 \Omega \cdot \mathrm{cm}^{-2}$ for $\operatorname{NDBP}(\mathrm{p}=0.002)$ (figure $3 \mathrm{c}$ ).

\section{Effects of exosomes on tight junctions}

Immunohistochemistry assessments confirmed that exosomes incubated with hMVEC-d monolayers induce disruption of cell membrane integrity, as illustrated by the altered topographic distribution of the tight junction protein ZO-1 (figure 4). We found that exosomes from NDBP and RDBP groups disrupted
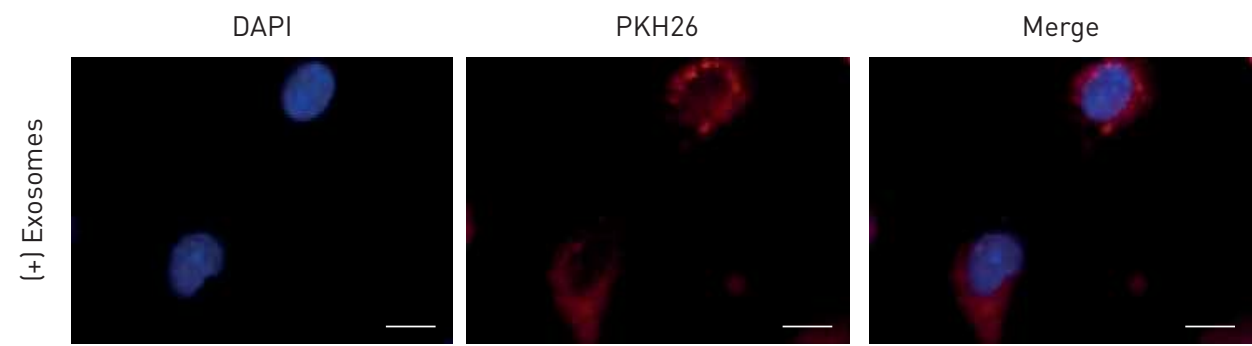

RDBP
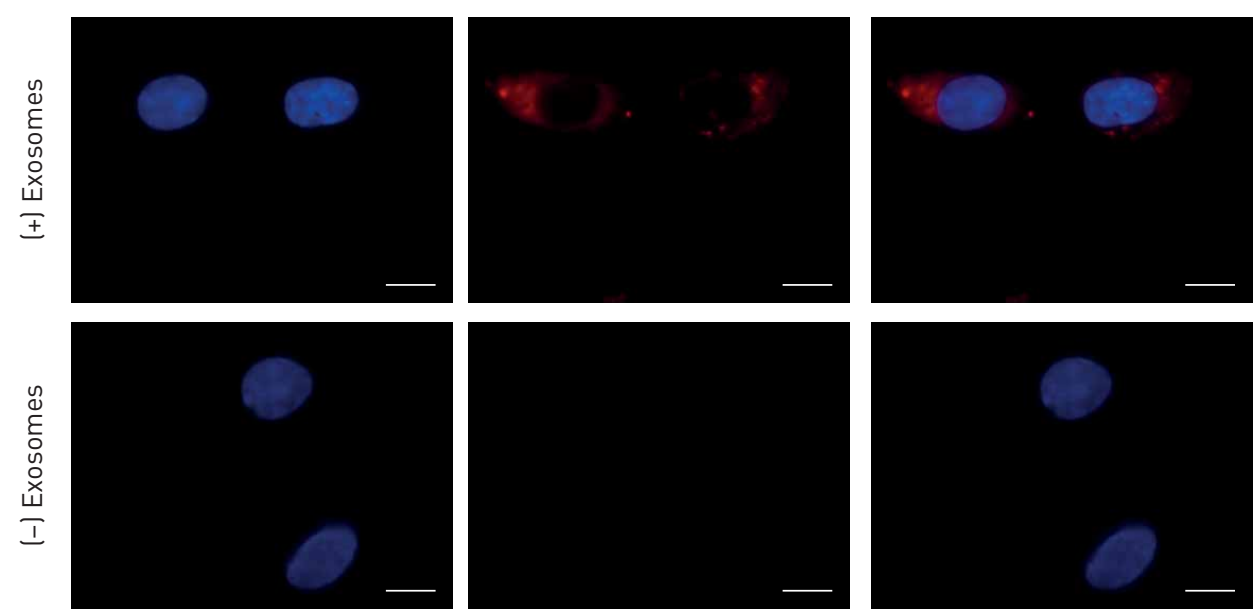

FIGURE 2 Confocal microscope images illustrating exosome uptake by naive human endothelial cells. DAPI: 4',6-diamidino-2-phenylindole; NDBP: normal dipping blood pressure; RDBP: reverse dipping blood pressure. Exosomes were isolated from plasma of NDBP and RDBP subjects and labelled with PKH26. Human endothelial cells were grown on coverslips for $24 \mathrm{~h}$ and the labelled exosomes derived from NDBP or RDBP with PKH26 were added to the cells at $37^{\circ} \mathrm{C}$ for $24 \mathrm{~h}$. Cells were washed and nuclei (blue) stained with DAPI. As controls, no exosomes were used, but PKH26 was added. Exosome uptake was measured using confocal laser scanning microscopy (Leica SP5; Leica Microsystems, Buffalo Grove, IL, USA) at $490 \mathrm{~nm}$ excitation and $502 \mathrm{~nm}$ emission. $\mathrm{n}=6$. Scale bar: $10 \mu \mathrm{m}$. 

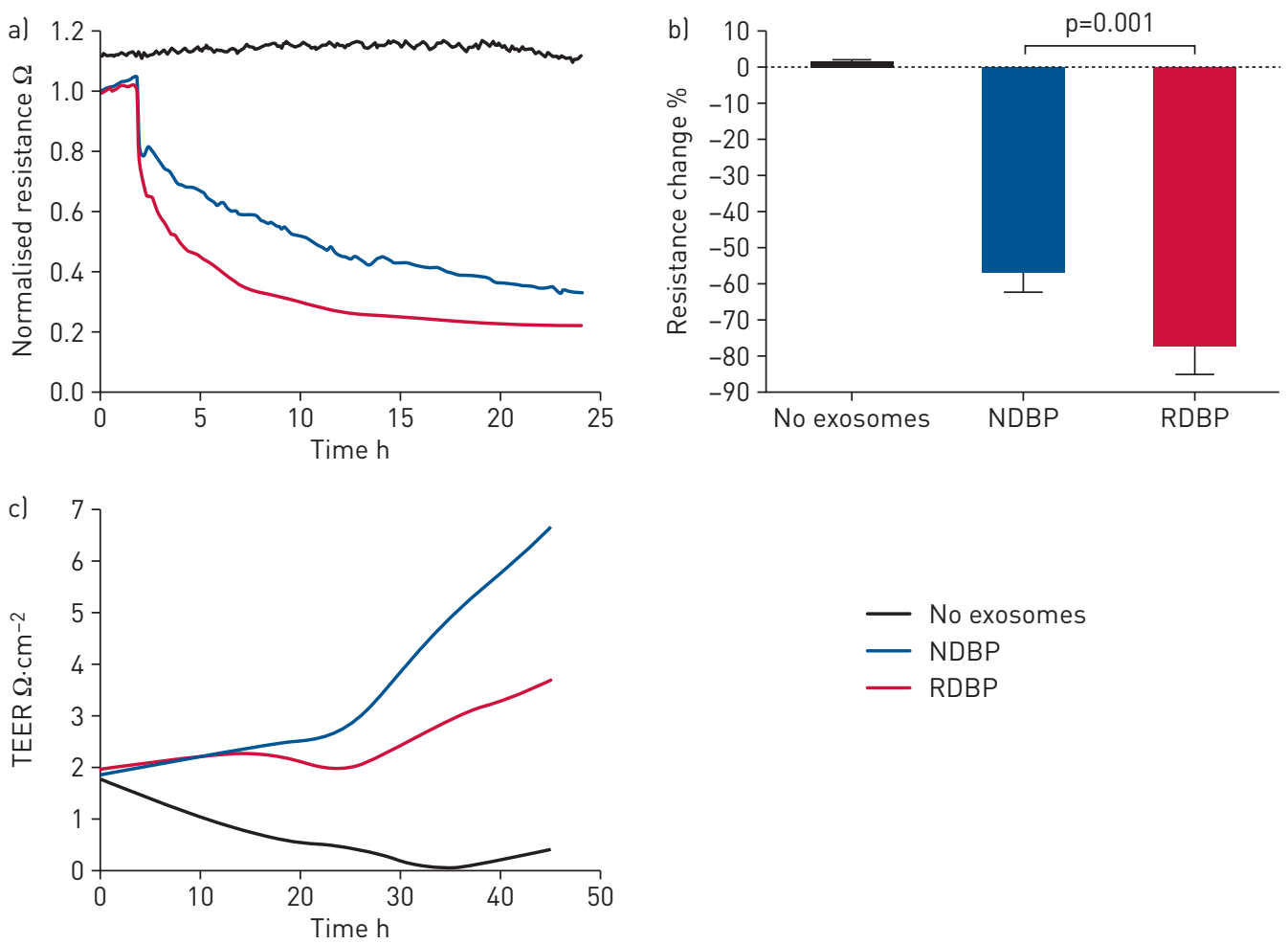

FIGURE 3 Effects of exosomes derived from reverse dipping blood pressure (RDBP) and normal dipping blood pressure (NDBP) subjects on endothelial cell monolayer resistance in vitro using two different approaches. TEER: trans-endothelial electrical resistance; ECIS: electric cell-substrate impedance sensing. First, the ECIS device was used to monitor barrier integrity continuously. a) Ensemble-averaged curves of ECIS-measured endothelial cell barrier resistance changes over time after administration of exosomes from adult patients with NDBP and RDBP compared with endothelial cells incubated with plasma-free media and empty exosomes. b) Evaluation of ECIS-measured endothelial cell barrier resistance changes of all 15 individual patients per group after exosome administration. The quantification averages of the disruption of endothelial cell monolayer resistance for all 15 subjects per group were $-78 \pm-7.2 \%$ (RDBP) compared with $-57 \pm-5.3 \%$ (NDBP) ( $p=0.001$ ). Secondly, endothelial cells were grown on transwell membranes to measure the integrity and permeability of the monolayer cells. c) Continuous measurement of cell monolayer barrier function (TEER) using membrane inserts in multiple wells to measure the resistance across the transwell membrane over time. TEER values for the average of each group: no exosomes, NDBP and RDBP. $n=15$.

endothelial cell tight junctions compared with untreated cells. Furthermore, exosomes derived from the RDBP group showed greater disruption of endothelial cell tight junctions compared with the NDBP group (figure 4a). STED super-resolution microscopy showed a very high magnification of ZO-1 immunoreactivity (figure $4 \mathrm{~b}$ ), allowing for visual identification of intact endothelial cell tight junctions versus disrupted tight junctions and the adhesion junctions between two cells. ZO-1 was linearly distributed in untreated cells, suggesting that exosomes mediate the disruption of tight junctions between cells. ZO-1 is a central regulator of VE-cadherin-dependent endothelial junctions that organise the cytoskeleton, tune cell-cell tension and control barrier formation [31]. Thus, we studied in vitro VE-cadherin protein expression on coverslips using naive human endothelial cells. We found that plasma exosomes from NDBP and RDBP samples incubated on naive endothelial cells diminished VE-cadherin adhesion junctions and tight junctions compared with untreated cells. The endothelial cell monolayer breakdown, as shown by a decrease in VE-cadherin, was observed only when exosomes derived from RDBP were used (figure 5a).

Since ICAM-1 is critical for the firm arrest and transmigration of leukocytes from blood vessels, we examined the effect of exosomes by ICAM-1 expression on barrier function (figure $5 \mathrm{~b}$ ). We found that immunostaining for ICAM-1 was weaker in untreated endothelial cells compared with endothelial cells treated with exosomes derived from the NDBP or RDBP groups.

\section{Exosomal miRNA profiling and validation}

Using human miRNA arrays, we identified 87 unique exosomal miRNAs that were detectable above the background level (figure 6a and b, and supplementary figure S2). Principal component analysis (PCA) and 
a)
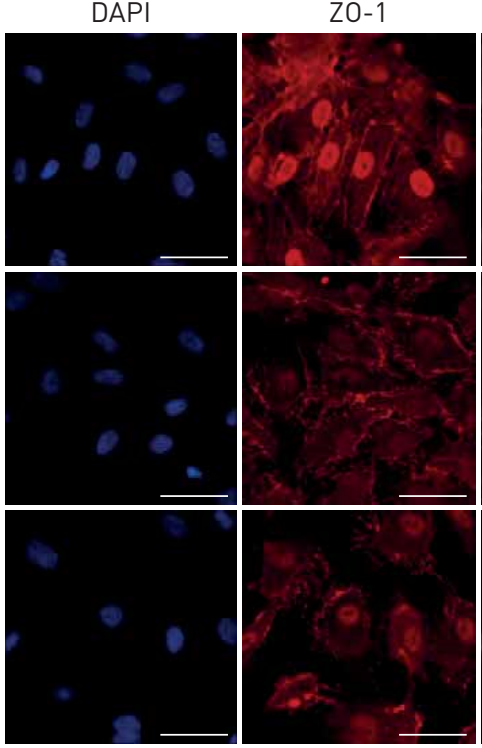
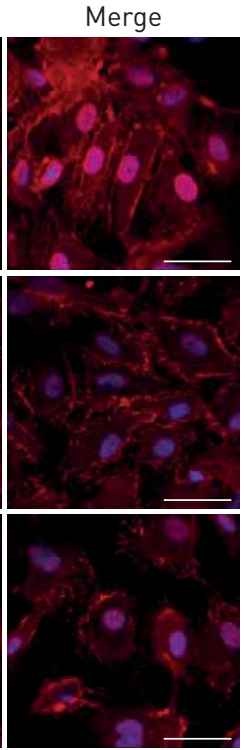

b)

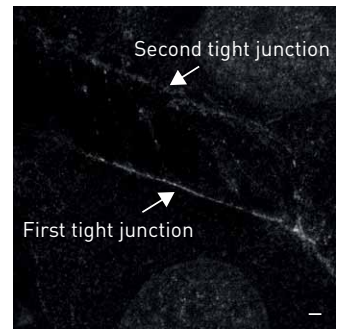

Untreated sample

NDBP

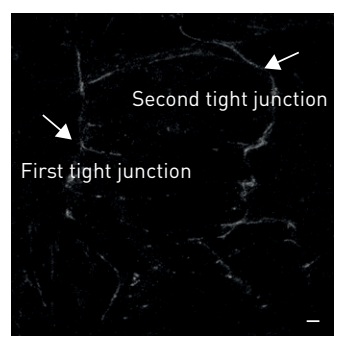

Treated with exosomes

FIGURE 4 Effect of plasma exosomes on naive endothelial cell zonula occludens (ZO)-1 tight junctions. DAPI: 4',6-diamidino-2-phenylindole; NDBP: normal dipping blood pressure; RDBP: reverse dipping blood pressure. Human endothelial cell monolayers were treated with exosomes derived from NDBP and RDBP subjects for $24 \mathrm{~h}$. Labelling was carried out as described in the Materials and methods. a) Representative images of at least eight separate experiments illustrating exosome-induced changes in expression of Z0-1. $n=8$. Scale bar: $10 \mu \mathrm{m}$. b) Stimulated emission depletion microscopy-derived image for ZO-1. As opposed to the intact tight junction of the cells not treated with exosomes, the tight junction integrity is markedly disrupted between two neighbouring cells after exosomes from RDBP are added to the media. $\mathrm{n}=8$. Scale bar: $500 \mu \mathrm{m}$.

a)
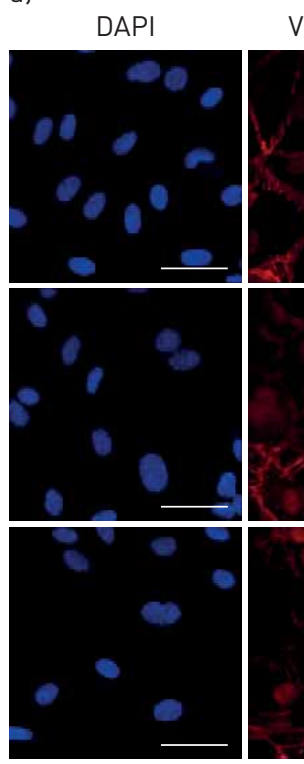

VE-
7
4
4
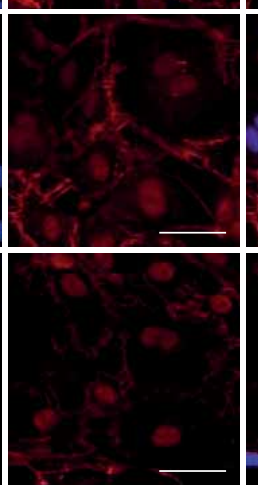

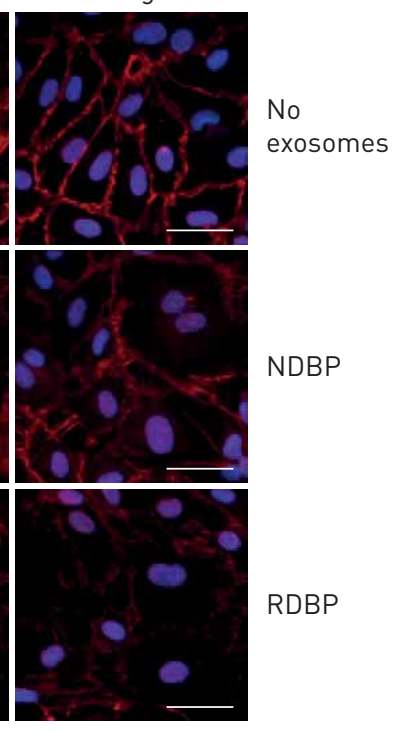

Merge b)

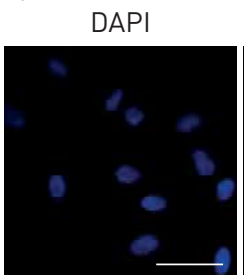

ICAM-1
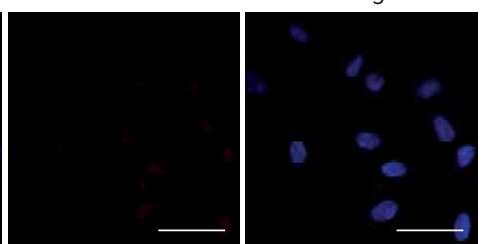

No

exosomes
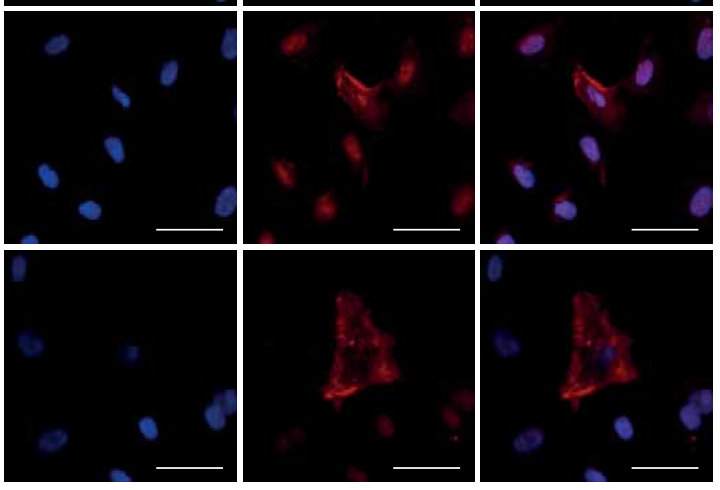

NDBP

RDBP

FIGURE 5 Effects of exosomes derived from reverse dipping blood pressure (RDBP) and normal dipping blood pressure (NDBP) subjects on tight junctions and adhesion molecule expression in naive primary human endothelial cells. DAPI: 4',6-diamidino-2-phenylindole; ICAM: intracellular adhesion molecule. a) Representative images of exosome-induced changes in expression of VE-cadherin adherens junctions in primary human endothelial cells after treatment with exosomes from RDBP and NDBP subjects. VE-cadherin protein is shown in red and nuclei are stained with DAPI (blue). Disruption of adherens junctions and tight junctions is more apparent in cells treated with exosomes from RDBP compared with NDBP subjects. b) Representative images of ICAM-1 immunohistochemistry in naive human primary endothelial cells following treatment with exosomes from RDBP and NDBP subjects. Exosomes from RDBP subjects induced increased ICAM-1 expression compared with NDBP subjects. ICAM-1 is shown in red and nuclei are stained with DAPI (blue). Images are representative of n=8 per group. Scale bar: $10 \mu \mathrm{m}$. 
a)

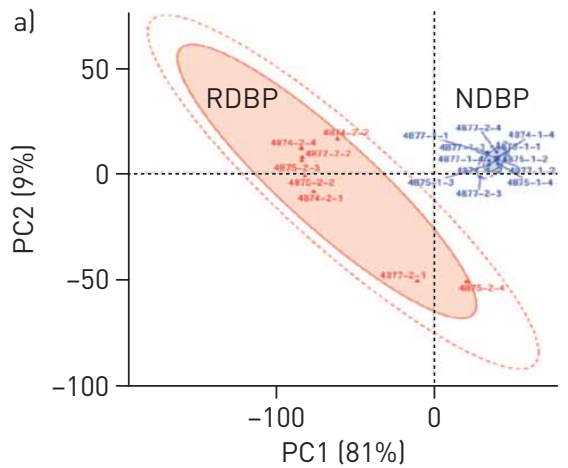

b)

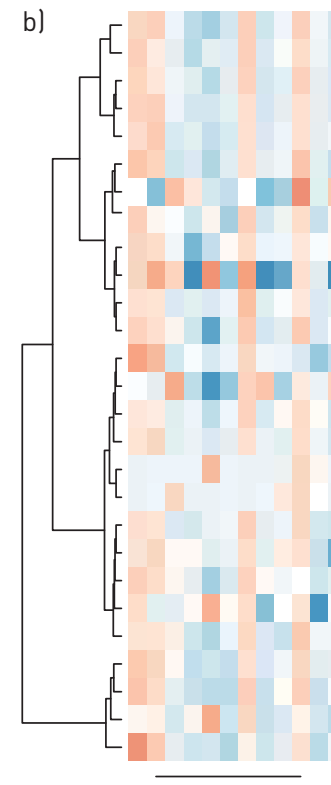

NDBP

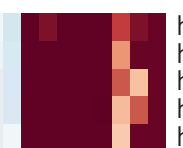

hsa-miR-6800-3p hsa-miR-1234-3p hsa-miR-6069 hsa-miR-3162-3p hsa-miR-1228-3p hsa-miR-1238-3p hsa-let-7f-1-3p hsa-miR-1281

hsa-miR-6797-3p hsa-miR-4652-3p hsa-miR-6737-3p hsa-miR-6508-3p hsa-miR-933 hsa-let-7b-3p hsa-let-7b-3p
hsa-miR-6515-3p hsa-miR-4515 hsa-miR-6834-3p hsa-miR-6795-3p hsa-miR-4649-3p hsa-miR-33b-3p hsa-miR-5571-5p hsa-miR-6813-3p hsa-miR-4725-5p hsa-miR-4665-3p hsa-miR-1304-3p hsa-miR-4313 hsa-miR-940
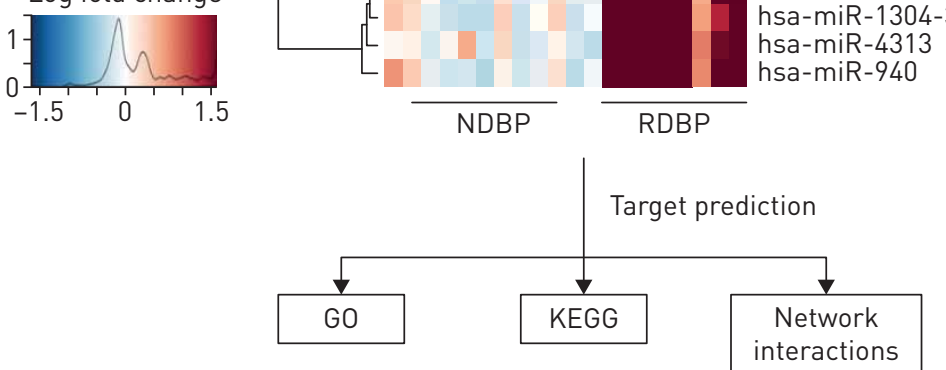

FIGURE 6 Plasma-derived exosome microRNA (miRNA) profiling. PCA: principal component analysis; NDBP: normal dipping blood pressure; RDBP: reverse dipping blood pressure; GO: Gene Ontology; KEGG: Kyoto Encyclopedia of Genes and Genomes. a) PCA plot of exosomal miRNAs from RDBP and NDBP subjects. PCA clustering of miRNA expression by subject group is denoted by different colours, and reveals complete and accurate separation of miRNA expression across the subjects from the two groups. b) Heatmap illustrating miRNA expression patterns in exosomes (dark red: increased miRNA expression; light blue: reduced miRNA expression). The dendrograms show hierarchical clustering representing the similarities and dissimilarities in expression profiles among individuals and miRNAs. In silico target gene predictions were used for GO, KEGG pathways and gene network interactions.

heatmap clustering revealed consistent group separation and categorical assignments concordant with the experimental groups, and showed substantial differences in miRNA expression profiles (figure $6 \mathrm{a}$ and $\mathrm{b}$ ). We found that 27 out of the 87 miRNAs were significantly upregulated in RDBP-derived exosomes (figure 6b). We validated four of these differentially expressed miRNAs using qRT-PCR. Furthermore, we validated four miRNAs overexpressed in RDBP compared with NDBP patients (figure 6b): hsa-miR-1234-3p (1.86 \pm 0.14 fold difference; $p=0.001)$, hsa-miR-1304-3p (1.91 \pm 0.11 fold difference; $p=0.003)$, hsa-miR-4313 $(2.01 \pm 0.18$ fold difference; $\mathrm{p}=0.001)$ and hsa-miR-940 $(2.19 \pm 0.17$ fold difference; $\mathrm{p}=0.001)$. These validated miRNAs showed the same trends as the expression differences identified in the miRNA arrays.

\section{Gene targets prediction}

We used several computational databases for the target predictions of 27 miRNAs and identified 5288 individual genes. To evaluate the biological role of the differentially expressed miRNA target genes, we performed a Gene Ontology (GO) classification enrichment analysis. Genes that showed a significance level $\mathrm{p}<0.01$ were selected and tested against the background set of all genes with GO annotation using established computational algorithms. GO revealed concordant pathways associated with vascular dysfunction (figure $7 \mathrm{a}$ and supplementary table $\mathrm{S} 1$ ).

We also performed Kyoto Encyclopedia of Genes and Genomes (KEGG) pathways enrichment analysis for differentially expressed miRNA target genes. The significant KEGG pathways and their genes are shown in table 2. Here, we highlight some of the pathways with potential biological significance in OSA, such as the AMP-activated protein kinase (AMPK) signalling pathway $(\mathrm{p}=1.65 \mathrm{E}-05)$, Ras signalling pathway ( $\mathrm{p}$-value=2.05E-05), cAMP signalling pathway $(\mathrm{p}=5.53 \mathrm{E}-05)$, phosphatidylinositol 3-kinase $(\mathrm{PI} 3 \mathrm{~K}) / \mathrm{Akt}$ signalling pathway $(\mathrm{p}=5.63 \mathrm{E}-04)$, insulin secretion $(\mathrm{p}=0.002)$, insulin resistance $(\mathrm{p}=0.023)$ and hypoxia inducible factor (HIF)-1 signalling pathway $(\mathrm{p}=0.024)$. 
a)

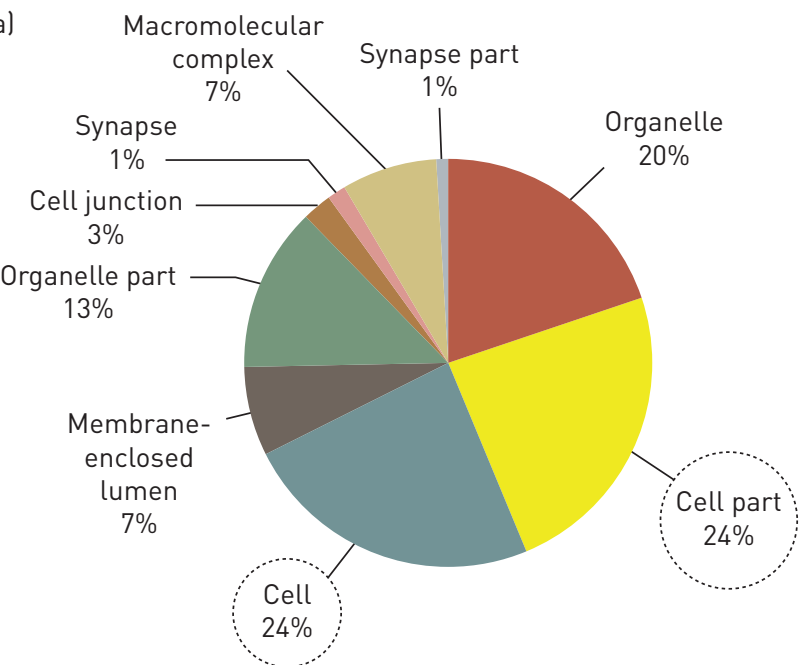

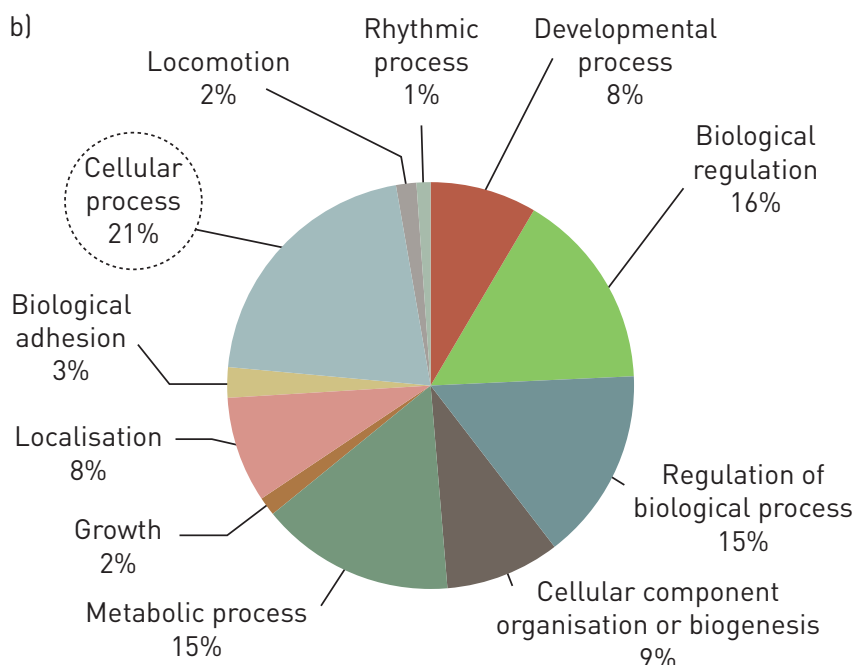

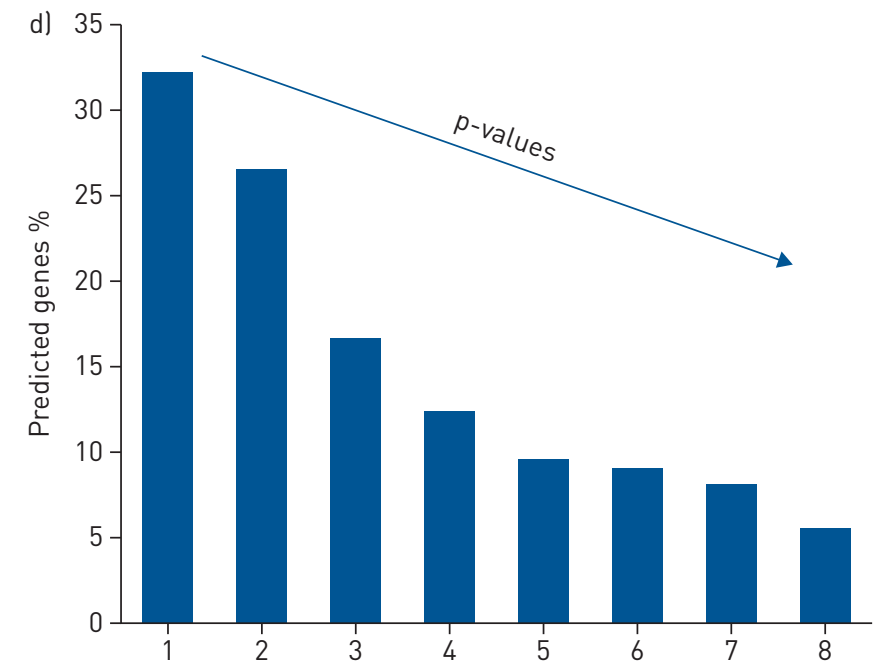

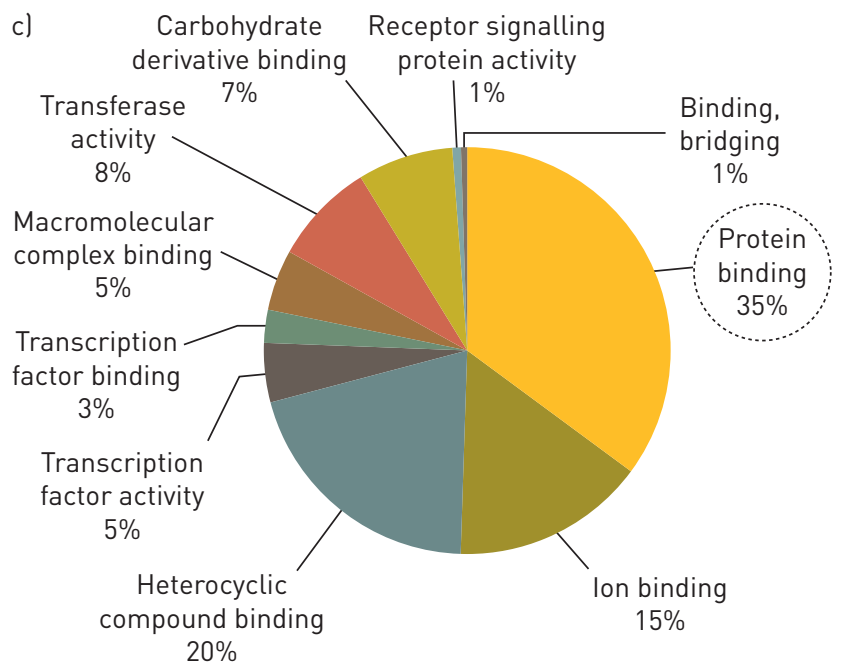

FIGURE 7 Gene Ontology (GO) for the differentially expressed microRNAs (miRNAs) in exosomes derived from reverse dipping blood pressure (RDBP) and normal dipping blood pressure (NDBP) subjects. GO analysis was used to assess a) cellular components, b) biological processes and c) molecular functions. The classification shows the top ranked significant GO terms. d) Gene target prediction genes derived from differentially expressed miRNAs in RDBP compared with NDBP subjects and their potential involvement in human disease. 1: metabolic; 2: cardiovascular; 3: neurological; 4: psychiatric; 5: haematological; 6: developmental; 7: renal; 8: ageing. The highest significance values were for metabolic and cardiovascular genes.

\section{Gene networks}

We constructed gene networks for predicted target genes involved in the pathways shown in table 2. Based on potential biological significance, we constructed two pathways: 1) for insulin resistance (figure 8a) [32-34] and 2) for HIF-1 signalling pathway (figure 8b) [35].

The Regulatory Network of Target Genes was constructed using STRING (http://string-db.org). In this network, several genes are ranked as highly significant in insulin resistance, e.g. SLC2A4, AKT3, CPT1B, CREB1 and CREB3L1, while in HIF-1 signalling the genes with high statistical significance were AKT3, ANGPT2, BCL2, CAMK2A, CAMK2G, CDKN1B and CREBBP.

\section{Discussion}

In this study, we found that RDBP in OSA alters functional properties of plasma exosomes, which are more disruptive of the permeability of the endothelial barrier, reduce ZO-1 and VE-cadherin, and increase ICAM-1 expression. Furthermore, the exosomes carry differentially expressed miRNAs in RDBP patients that regulate potentially important genomic pathways such as HIF-1 signalling and insulin resistance. Conceivably, these changes promote vascular damage in vivo, and underlie endothelial dysfunction and abnormal circadian blood pressure regulation in OSA. 
TABLE 2 Kyoto Encyclopedia of Genes and Genomes (KEGG) pathways identified in target predication genes that were found in differentially expressed microRNAs in reverse dipping blood pressure versus normal dipping blood pressure

\begin{tabular}{lcc} 
Terms & KEGG pathway & p-value \\
\hline hsa04152 & AMPK signalling pathway\# & $1.65 \mathrm{E}-05$ \\
hsa04014 & Ras signalling pathway & \\
hsa04024 & cAMP signalling pathway & $2.05 \mathrm{E}-05$ \\
hsa05231 & Choline metabolism in cancer & $5.53 \mathrm{E}-05$ \\
hsa04520 & Adherens junction & $1.04 \mathrm{E}-04$ \\
hsa04068 & FoxO signalling pathway & $1.13 \mathrm{E}-04$ \\
hsa04012 & ErbB signalling pathway & $1.46 \mathrm{E}-04$ \\
hsa04713 & Circadian entrainment & $1.53 \mathrm{E}-04$ \\
hsa04010 & MAPK signalling pathway & $2.51 \mathrm{E}-04$ \\
hsa04151 & PI3K-Akt signalling pathway & $4.54 \mathrm{E}-04$ \\
hsa04911 & Insulin secretion & $5.63 \mathrm{E}-04$ \\
hsa04310 & Wnt signalling pathway & 0.00225 \\
hsa04350 & TGF- $\beta$ signalling pathway & 0.00397 \\
hsa04931 & Insulin resistance & 0.00724 \\
hsa04066 & HIF-1 signalling pathway & 0.023119 \\
\end{tabular}

AMPK: AMP-activated protein kinase; MAPK: mitogen-activated protein kinase; PI3K: phosphatidylinositol 3-kinase; TGF: transforming growth factor; HIF: hypoxia inducible factor. \#: pathways with potential biological significance in obstructive sleep apnoea.

Exosomes are released from various tissues and carry a specifically sorted cargo of signalling molecules, including miRNAs for intercellular communication and regulation of organ function $[12,14,36,37]$. Dysregulation of the miRNA content in the exosomes can provide valuable insights into disease mechanisms and candidate biomarkers [38,39]. We have shown that exosomes induce in vitro endothelial
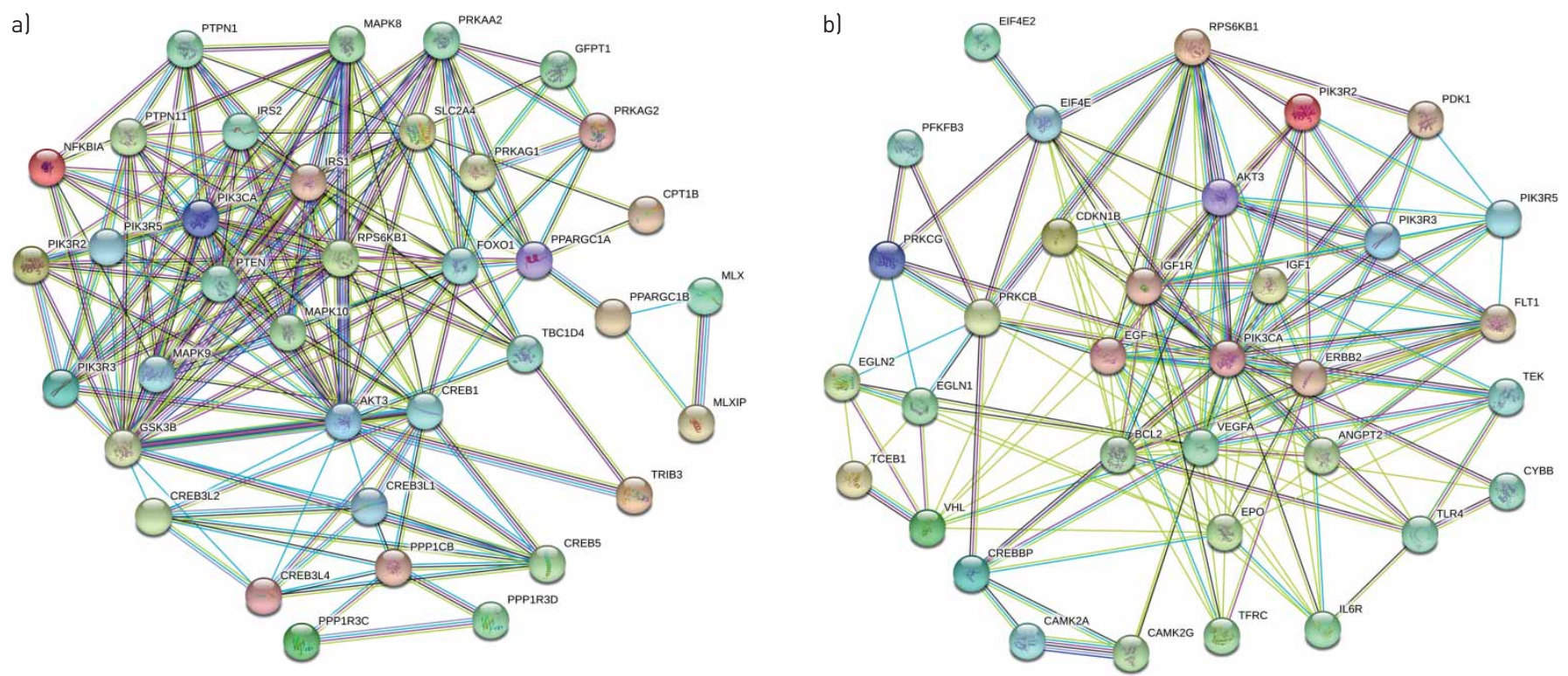

Known interactions:

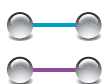

From curated databases

Experimentally determined
Predicted interactions:

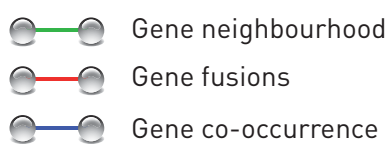

Others:

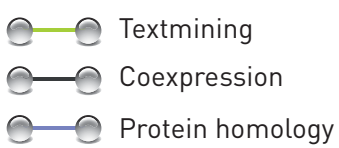

FIGURE 8 Gene network representation of interactions between gene target predictions of exosomal miRNAs derived from reverse dipping blood pressure compared with normal dipping blood pressure that involve a) insulin resistance and b) hypoxia inducible factor- 1 signalling pathways as shown in table 2. 
dysfunction in healthy humans exposed to acute intermittent hypoxia [20] and reported similar findings in severe OSA patients with obesity hypoventilation [21].

The integration, transmission and regulation of mechanical forces at sites of adhesion in the endothelium drive the progression of hypertension [40]. Interendothelial cell junctions include adherens junctions and tight junctions, which play pivotal roles in tissue integrity, barrier function and cell-cell communication [41]. The disruption of tight junctions leads to increased paracellular permeability and endothelial dysfunction [42]. Previously, we have shown that exosomes from children [19] and adults [21] with untreated OSA disrupt ZO-1 tight junctions compared with either healthy controls or effectively treated patients. In this study, we focused on the effects of exosomes, derived from a particularly high-risk phenotype (i.e. RDBP), on endothelial barrier function [19, 43, 44]. RDBP exosomes reduced ZO-1 and VE-cadherin expression and increased ICAM-1 expression when delivered to naive endothelial cells compared with NDBP exosomes. Thus, exosomes from RDBP patients exhibit pathophysiological properties that promote disruption of tight junctions and adherens junctions. VE-cadherin in endothelial cell adherens junctions maintains the integrity of tight junction activity [41]. ZO-1 is a junctional adaptor protein that interacts with other junctional components to stabilise the tight junction intercellular interface [45]. Finally, ICAM-1 transmembrane protein is constitutively expressed in low concentrations in leukocytes and endothelial cells, but with increased expression, it facilitates the slowing, rolling and activation of macrophages to cross the endothelial junctions and foster matrix changes associated with vascular stiffness. Thus, the increases in ICAM-1 elicited by RDBP exosomes reflect an enhanced propensity for the initiation of vascular stiffness $[12,20]$. Furthermore, RDBP exosomes induced changes in endothelial permeability compared with NDBP exosomes through the loosening of adherens junctions (ECIS measurements). Thus, the exosomal cargo and its structural and functional effects on the endothelium differ between RDBP and NDBP patients, providing inferential assumptions about mechanisms underlying the onset and progression of nocturnal hypertension in OSA.

Previously, we compared the effects of continuous positive airway pressure (CPAP) treatment of OSA on plasma miRNA expression patterns in OSA patients with resistant hypertension. Two groups were compared: favourable response to CPAP versus nonsignificant reduction in blood pressure despite adherent CPAP treatment ( $24 \mathrm{~h}$ mean blood pressure reduction $\geqslant-4.5$ versus $<-4.5 \mathrm{mmHg}$ ) [7]. Here, we identified a cluster of five circulating miRNAs that enabled accurate prediction of significant blood pressure response to CPAP. Furthermore, we have identified several exosomal miRNA candidates that differentiate children with OSA or with obesity and endothelial dysfunction from matched children without endothelial dysfunction, and demonstrated the mechanistic role of one such miRNA in reproducing endothelial dysfunction both in vitro and in murine models [19]. In the current study, we found a selected cluster of 27 miRNAs to be upregulated in RDBP compared with matched NDBP patients, suggesting that these miRNAs play a role in nocturnal hypertension in OSA. To further understand the functional significance of the upregulated exosomal miRNAs in RDBP, we examined their gene targets using multiple in silico bioinformatic tools. Although miRNAs generally influence the expression of their target genes negatively via mRNA degradation and translational repression, there is evidence that they may also enhance the expression of genes [46]. To avoid an excessive number of genes predicted by single bioinformatics approaches, we applied a strict curation of such lists through combinatorial bioinformatic analyses and found that $15 \%$ of the target genes were involved in cardiometabolic processes. We showed that $32 \%$ of the predicted target genes are associated with metabolism and $27 \%$ with CVD. Important pathways significantly altered in RDBP include the AMPK signalling pathway, Ras signalling pathway, cAMP signalling pathway, adherens junction, circadian entrainment, mitogen-activated protein kinase signalling pathway, PI3K-Akt signalling pathway, insulin secretion, Wnt signalling pathway, transforming growth factor- $\beta$ signalling pathway, insulin resistance and HIF-1 signalling pathway. All of these pathways have been implicated in CVD. Therefore, further studies examining the specific pathways in RDBP patients and their responses to treatment, as well as murine models targeting these pathways through selective alterations of exosomal miRNA cargo, will provide mechanistic insights and potential therapeutic targets.

Some strengths and limitations are worth noting in the present study. First, our patients were carefully phenotyped as RDBP or NDBP and matched for potential confounders. However, this proof-of-concept study does not include a normotensive OSA control group and, due to the cross-sectional design, causal inferences are not possible. Our in vitro reporter studies do not demonstrate that nocturnal dipping patterns will be altered in vivo by the exosomal miRNAs that are differentially expressed in the RDBP circulating exosomes [14]. The transfer and translation of exosomal mRNA and miRNA in target cells to suppress or enhance gene expression is a breakthrough in exosome biology $[18,19,37]$. The selectivity of miRNA loading into exosomes and their stability prompted our search for potential differences in miRNA exosomal cargo using microarray approaches [19, 20, 47, 48]. However, it is possible that additional 
constitutive exosome cargo components such as proteins, RNAs, DNAs or lipids play a functional role, which should be explored in future studies. The restricted quantities of plasma that were available from the participants were constrained by requirements of the bioethical committee and precluded exploration of differences in miRNA exosomal cargo in a cell-source-specific manner. In addition, the lack of quantification of the immunostaining signal and ICAM mRNA expression in our samples are salient limitations. These limitations should be considered in future validation studies.

\section{Conclusions}

Exosomes derived from OSA with RDBP enhance disruption of endothelial cell monolayer tight junctions and increase endothelial dysfunction. A selective cluster of miRNAs regulates relevant biological pathways that underlie endothelial dysfunction and RDBP in OSA. We propose that a unique set of circulating exosomal miRNAs plays a mechanistic role in nocturnal hypertension associated with OSA. Thus, exosomal miRNAs may serve as both biomarkers and therapeutic targets in OSA-related hypertension.

Conflict of interest: None declared.

Support statement: This work was supported by the US Dept of Veterans Affairs grant VA CSR\&D; 1IK2CX001026-01. Funding information for this article has been deposited with the Crossref Funder Registry.

\section{References}

$1 \quad$ Malhotra A, White DP. Obstructive sleep apnoea. Lancet 2002; 360: 237-245.

2 Nieto FJ, Young TB, Lind BK, et al. Association of sleep-disordered breathing, sleep apnea, and hypertension in a large community-based study. Sleep Heart Health Study. JAMA 2000; 283: 1829-1836.

3 Morrell MJ, Finn L, Kim H, et al. Sleep fragmentation, awake blood pressure, and sleep-disordered breathing in a population-based study. Am J Respir Crit Care Med 2000; 162: 2091-2096.

4 Kuniyoshi FH, Garcia-Touchard A, Gami AS, et al. Day-night variation of acute myocardial infarction in obstructive sleep apnea. J Am Coll Cardiol 2008; 52: 343-346.

5 Manfredini R, Boari B, Salmi R, et al. Twenty-four-hour patterns in occurrence and pathophysiology of acute cardiovascular events and ischemic heart disease. Chronobiol Int 2013; 30: 6-16.

6 Sasaki N, Ozono R, Edahiro Y, et al. Impact of non-dipping on cardiovascular outcomes in patients with obstructive sleep apnea syndrome. Clin Exp Hypertens 2015; 37: 449-453.

7 Sanchez-de-la-Torre M, Khalyfa A, Sanchez-de-la-Torre A, et al. Precision medicine in patients with resistant hypertension and obstructive sleep apnea: blood pressure response to continuous positive airway pressure treatment. J Am Coll Cardiol 2015; 66: 1023-1032.

8 Javaheri S, Barbe F, Campos-Rodriguez F, et al. Sleep apnea: types, mechanisms, and clinical cardiovascular consequences. J Am Coll Cardiol 2017; 69: 841-858.

9 Munoz-Hernandez R, Vallejo-Vaz AJ, Sanchez Armengol A, et al. Obstructive sleep apnoea syndrome, endothelial function and markers of endothelialization. Changes after CPAP. PLoS One 2015; 10: e0122091.

10 Schwarz EI, Puhan MA, Schlatzer C, et al. Effect of CPAP therapy on endothelial function in obstructive sleep apnoea: a systematic review and meta-analysis. Respirology 2015; 20: 889-895.

11 Thery C, Ostrowski M, Segura E. Membrane vesicles as conveyors of immune responses. Nat Rev Immunol 2009; 9: 581-593.

12 Khalyfa A, Kheirandish-Gozal L, Gozal D. Circulating exosomes in obstructive sleep apnea as phenotypic biomarkers and mechanistic messengers of end-organ morbidity. Respir Physiol Neurobiol 2018; 256: 143-156.

13 Khalyfa A, Kheirandish-Gozal L, Gozal D. Exosome and macrophage crosstalk in sleep-disordered breathing-induced metabolic dysfunction. Int J Mol Sci 2018; 19: E3383.

14 Khalyfa A, Gozal D. Exosomal miRNAs as potential biomarkers of cardiovascular risk in children. $J$ Transl Med 2014; 12: 162 .

15 de Jong OG, Verhaar MC, Chen Y, et al. Cellular stress conditions are reflected in the protein and RNA content of endothelial cell-derived exosomes. J Extracell Vesicles 2012; 1: 18396.

16 Borosch S, Dahmen E, Beckers C, et al. Characterization of extracellular vesicles derived from cardiac cells in an in vitro model of preconditioning. J Extracell Vesicles 2017; 6: 1390391

17 Simons M, Raposo G. Exosomes - vesicular carriers for intercellular communication. Curr Opin Cell Biol 2009; 21: 575-581.

18 Valadi H, Ekstrom K, Bossios A, et al. Exosome-mediated transfer of mRNAs and microRNAs is a novel mechanism of genetic exchange between cells. Nat Cell Biol 2007; 9: 654-659.

19 Khalyfa A, Kheirandish-Gozal L, Khalyfa AA, et al. Circulating plasma extracellular microvesicle microRNA cargo and endothelial dysfunction in children with obstructive sleep apnea. Am J Respir Crit Care Med 2016; 194: $1116-1126$.

20 Khalyfa A, Zhang C, Khalyfa AA, et al. Effect on intermittent hypoxia on plasma exosomal micro RNA signature and endothelial function in healthy adults. Sleep 2016; 39: 2077-2090.

21 Bhattacharjee R, Khalyfa A, Khalyfa AA, et al. Exosomal cargo properties, endothelial function and treatment of obesity hypoventilation syndrome: a proof of concept study. J Clin Sleep Med 2018; 14: 797-807.

22 Iaconetti C, Sorrentino S, De Rosa S, et al. Exosomal miRNAs in heart disease. Physiology 2016; 31: 16-24.

23 Khalyfa A, Kheirandish-Gozal L, Khalyfa AA, et al. Circulating plasma extracellular microvesicle miRNA cargo and endothelial dysfunction in OSA children. Am J Respir Crit Care Med 2016; 194: 1116-1126.

24 Khalyfa A, Khalyfa AA, Akbarpour M, et al. Extracellular microvesicle microRNAs in children with sickle cell anemia with divergent clinical phenotypes. Br J Haematol 2016; 174: 786-798.

25 Khalyfa A, Youssefnia N, Foster GE, et al. Plasma exosomes and improvements in endothelial function by angiotensin 2 type 1 receptor or cyclooxygenase 2 blockade following intermittent hypoxia. Front Neurol 2017; 8: 709. 

live-cell microscopy. I Cell Biochem 2010; 111: 488-496. organ-on-a-chip microsystems. Lab Chip 2015; 15: 745-752.

28 Khalyfa A, Capdevila OS, Buazza MO, et al. Genome-wide gene expression profiling in children with non-obese obstructive sleep apnea. Sleep Med 2009; 10: 75-86.

29 Giaever I, Keese CR. Use of electric fields to monitor the dynamical aspect of cell behavior in tissue culture. IEEE Trans Biomed Eng 1986; 33: 242-247.

30 Keese CR, Bhawe K, Wegener J, et al. Real-time impedance assay to follow the invasive activities of metastatic cells in culture. Biotechniques 2002; 33: 842-844.

31 Tornavaca O, Chia M, Dufton N, et al. ZO-1 controls endothelial adherens junctions, cell-cell tension, angiogenesis, and barrier formation. J Cell Biol 2015; 208: 821-838.

32 King GL, Park K, Li Q. Selective insulin resistance and the development of cardiovascular diseases in diabetes: the 2015 Edwin Bierman Award Lecture. Diabetes 2016; 65: 1462-1471.

33 Rask-Madsen C, Li Q, Freund B, et al. Loss of insulin signaling in vascular endothelial cells accelerates atherosclerosis in apolipoprotein E null mice. Cell Metab 2010; 11: 379-389.

34 Vicent D, Ilany J, Kondo T, et al. The role of endothelial insulin signaling in the regulation of vascular tone and insulin resistance. J Clin Invest 2003; 111: 1373-1380.

35 Abe H, Semba H, Takeda N. The roles of hypoxia signaling in the pathogenesis of cardiovascular diseases. J Atheroscler Thromb 2017; 24: 884-894.

36 Barile L, Vassalli G. Exosomes: therapy delivery tools and biomarkers of diseases. Pharmacol Ther 2017; 174: 63-78.

37 Kosaka N, Iguchi H, Yoshioka Y, et al. Secretory mechanisms and intercellular transfer of microRNAs in living cells. J Biol Chem 2010; 285: 17442-17452.

38 Iorio MV, Croce CM. Causes and consequences of microRNA dysregulation. Cancer J 2012; 18: $215-222$.

39 Kourembanas S. Exosomes: vehicles of intercellular signaling, biomarkers, and vectors of cell therapy. Annu Rev Physiol 2015; 77: 13-27.

40 Conway D, Schwartz MA. Lessons from the endothelial junctional mechanosensory complex. F1000 Biol Rep 2012; 4: 1 .

41 Wallez Y, Huber P. Endothelial adherens and tight junctions in vascular homeostasis, inflammation and angiogenesis. Biochim Biophys Acta 2008; 1778: 794-809.

42 Bazzoni G, Dejana E. Endothelial cell-to-cell junctions: molecular organization and role in vascular homeostasis. Physiol Rev 2004; 84: 869-901.

43 Heijink IH, Brandenburg SM, Noordhoek JA, et al. Characterisation of cell adhesion in airway epithelial cell types using electric cell-substrate impedance sensing. Eur Respir J 2010; 35: 894-903.

44 Bicker J, Alves G, Fortuna A, et al. Blood-brain barrier models and their relevance for a successful development of CNS drug delivery systems: a review. Eur J Pharm Biopharm 2014; 87: 409-432.

45 Van Itallie CM, Fanning AS, Bridges A, et al. ZO-1 stabilizes the tight junction solute barrier through coupling to the perijunctional cytoskeleton. Mol Biol Cell 2009; 20: 3930-3940.

46 Huntzinger E, Izaurralde E. Gene silencing by microRNAs: contributions of translational repression and mRNA decay. Nat Rev Genet 2011; 12: 99-110.

47 Vlassov AV, Magdaleno S, Setterquist R, et al. Exosomes: current knowledge of their composition, biological functions, and diagnostic and therapeutic potentials. Biochim Biophys Acta 2012; 1820: 940-948.

48 Ferrante SC, Nadler EP, Pillai DK, et al. Adipocyte-derived exosomal miRNAs: a novel mechanism for obesity-related disease. Pediatr Res 2015; 77: 447-454. 